\title{
Identifying compositional and structural changes in the nucleus pulposus from patients with lumbar disc herniation using Raman spectroscopy
}

\author{
XUEHUI WANG ${ }^{1,2 *}$, JIANFANG MENG ${ }^{3 *}$, TONGXING ZHANG ${ }^{4}$, WILLIAM WEIJIA LV ${ }^{5}$, \\ ZHAO LIANG ${ }^{6}$, QIAN SHI ${ }^{1},{\text { ZHAOYANG } \mathrm{LI}^{3} \text { and TAO ZHANG }}^{2}$ \\ ${ }^{1}$ First Central Clinical College, Tianjin Medical University, Tianjin 300070; ${ }^{2}$ Department of Orthopaedics, \\ Tianjin First Central Hospital, Tianjin 300192; ${ }^{3}$ School of Materials Science and Engineering, Tianjin University, \\ Tianjin 300350; ${ }^{4}$ Department of Orthopaedics, Tianjin Hospital, Tianjin Medical University, Tianjin 300211; \\ ${ }^{5}$ Department of Orthopaedics and Traumatology, The University of Hong Kong, Hong Kong, SAR; \\ ${ }^{6}$ Biobank, Tianjin First Central Hospital, Tianjin 300192, P.R. China
}

Received October 20, 2019; Accepted February 10, 2020

DOI: $10.3892 /$ etm.2020.8729

\begin{abstract}
Lower back pain (LBP) is one of the most common musculoskeletal complaints worldwide. Intervertebral disc degeneration (IDD) is considered to be a significant contributor to LBP; however, the mechanisms underlying IDD remain to be fully elucidated. One of the major features of IDD is the decreased content of type II collagen and proteoglycans in the nucleus pulposus (NP). The present study aimed to investigate the biochemical mechanisms of IDD at the microscopic level using Raman spectroscopy. Raman spectroscopy, based on inelastic scattering of light, is an emerging optical technique that may measure the chemical composition of complex biological samples, including biofluids, cells and tissues. In the present study, 30 NP tissue samples from 30 patients who were diagnosed with lumbar disc herniation and received spinal fusion surgery to relieve LBP were obtained and analyzed. Routine pre-operative 3.0T, T2-weighed MRI was used to classify the cases according to Pfirrmann grades and the T2 signal intensity value of the NP was measured. Subsequently, all NP samples were scanned and analyzed using a Laser MicroRaman Spectrometer at room temperature. The Raman spectral results demonstrated that the relative content of
\end{abstract}

Correspondence to: Dr Zhaoyang Li, School of Materials Science and Engineering, Tianjin University, 135 Yaguan Road, Tianjin 300350, P.R. China

E-mail: zyli@tju.edu.cn

Dr Tao Zhang, Department of Orthopaedics, Tianjin First Central Hospital, 24 Fukang Road, Tianjin 300192, P.R. China

E-mail: zht70@sina.com

${ }^{*}$ Contributed equally

Key words: lumbar disc herniation, Raman spectroscopy, magnetic resonance imaging, nucleus pulposus proteoglycans, expressed as the relative intensity ratio of two peaks $\left(\mathrm{I}_{1064} / \mathrm{I}_{1004}\right)$, was significantly inversely correlated with the Pfirrmann grade $(\rho=-0.6462 ; \mathrm{P}<0.0001)$, whereas the content of collagen (amide I) was significantly positively correlated with the Pfirrmann grade $(\rho=0.5141 ; \mathrm{P}<0.01)$. In conclusion, the higher relative intensity of the ratio of two peaks $\left(\mathrm{I}_{1670} / \mathrm{I}_{1640}\right.$; Amide I) represented a higher fractional content of disordered collagen, which suggested that the defective collagen structure may lead to NP abnormalities.

\section{Introduction}

Lower back pain (LBP) is one of the most common musculoskeletal complaints, with $\sim 84 \%$ of the population encountering LBP at a certain period during their lifetime (1). Although there are numerous potential sources of pain in the lumbar spine, intervertebral disc degeneration (IDD) is considered to be a major contributor to LBP (2). It is reported that $\sim 40 \%$ of LBP is of discogenic origin (3) and in the clinic, lumbar disc herniation (LDH) was observed to be a major contributor to LBP (4-6). Herniation, which refers to the displacement of intervertebral disc material beyond the normal margins of the disc space, was initially described as disc 'rupture' (1). In China, LDH has an incidence rate of $4.26 \%$, affecting $1.9-7.6 \%$ of males and 2.2-5.0\% of females (7).

MRI, with its high sensitivity to the tissue water content, has become an accurate tool for assessing IDD (8). Although the five-level Pfirrmann grading system is commonly used in clinical practice, there is a lack of information about biochemical changes that occur in the disc (9). At present, numerous quantitative MRI diagnostic methods have already been used to quantitatively explore the process of IDD, which include in vivo ${ }^{23} \mathrm{Na} \mathrm{MRI}$, quantitative high-resolution magic angle spinning nuclear MR spectroscopy, proton T2 imaging and T1rho imaging (10). However, these novel MRI techniques mainly monitor the concentration of proteoglycans and water in the discs and are not suited to evaluate the changes in collagen content and structure within the nucleus pulposus 
(NP). Furthermore, the requirements for tissue processing, including fixation, dehydration and staining, are major disadvantages of routine pathological and histological diagnostic methods due to the possibility of introducing artifacts (11).

Raman spectroscopy, based on inelastic scattering of light, is an emerging optical technique (12). It is widely used in the fields of medicine and life sciences due to its advantages of being label-free, non-invasive and an objective diagnostic tool at the molecular level. The advantage of a label-free technique is the ability to provide important information on the unmodified target molecules (13-15). Raman spectroscopy is able to detect biological macromolecules, including proteins, lipids, and DNA in biological samples and it provides abundant molecular information at the microscopic level (16). In recent years, the significant developments in Raman spectroscopic technology have facilitated it becoming a research hotspot for the early stages of disease and intra-operative diagnosis $(17,18)$.

To the best of our knowledge, the present study was the first to use Raman spectroscopy to identify compositional and structural changes in the NP from patients with LDH, in addition to exploring the biochemical mechanisms of IDD at the microscopic level.

\section{Materials and methods}

Patient samples. The study protocol was approved by the Ethics Review Board of Tianjin First Central Hospital (TFCH; Tianjin, China); the sample collection was approved by the Clinical Research Ethics Committee of TFCH and written informed consent was obtained from all patients. A total of $30 \mathrm{NP}$ samples were obtained from 30 patients (19 males and 11 females; age, 26-75 years; mean age, 51.7 years), who were diagnosed with LDH and received spinal fusion surgery to relieve LBP, between June 2016 and June 2018. Surgery is only appropriate in patients with nerve-root compression that is confirmed by CT or MRI, who present with a corresponding sciatica syndrome and have no response to 6 weeks of conservative therapy (1).

Preparation of NP samples. The patients' NP tissues were removed for treatment purposes during surgery and stored in a sterile plastic container with the patient's data. The NP samples were maintained on ice during transportation to the laboratory, which was completed within 30 min. NP samples were subsequently washed with physiological saline solution to remove the blood, sliced into $4-\mathrm{mm}^{2}$ thick sections and embedded in optimal cutting temperature medium (OCT) within a plastic mould, which was then frozen in liquid nitrogen. All frozen tissue blocks were cryosectioned to a thickness of $30 \mu \mathrm{m}$, mounted on glass coverslips and stored at $-20^{\circ} \mathrm{C}$ until required for further use. All procedures were performed in a sterile environment.

Pre-operative MRI measurements and assessment. All patients underwent a routine lumbar MRI scan prior to surgery. MRI imaging of the lumbar spine $\left(\mathrm{L}_{1} / \mathrm{L}_{2}\right.$ to $\left.\mathrm{L}_{5} / \mathrm{S}_{1}\right)$ was performed using T2-weighted sequences with a Siemens 3.0 T scanner (Siemens Healthineers) at the MRI center of TFCH. A T2-weighted imaging (T2WI) sequence was recorded using the following parameters: Axis T2WI repetition time (TR),
4,600 msec; echo time (TE), 94 msec; field of view (FOV), 210x210 mm; slice thickness, $4.0 \mathrm{~mm}$; scanning slice number, 3; Voxel size, $0.7 \times 0.5 \times 4.0 \mathrm{~mm}$; sagittal T2WI:TR, 3,000 msec; TE, $48 \mathrm{msec}$; FOV, 280x280 mm; slice thickness, $3.0 \mathrm{~mm}$; scanning slice number, 12; Voxel size, $1.2 \times 0.9 \times 3.0 \mathrm{~mm}$.

The grade of IDD was evaluated using T2-weighted images according to the Pfirrmann classification system (19). All MRI scans were reviewed independently and randomly by two experienced radiologists who were blinded to the clinical data and disagreements between the two observers were resolved by consensus following consultation with a third radiologist. After 3 months, the two observers evaluated all MRI images independently and randomly for a second time (the observers were not aware that they were evaluating the same images).

T2 signal intensity value measurement of $N P$. The T2 signal intensity value of the NP was measured using Siemens post-processing software on sagittal T2-weighted images. A single operator placed small, round regions of interest $\left(20-40 \mathrm{~mm}^{2}\right)$ within the anterior, middle and posterior positions of the NP on the target intervertebral disc. The recorded values were then averaged over three successive measurements at each selected location (8). The same method was used to measure the cerebrospinal fluid brightness value of the lumbar segment. The T2 signal intensity value of the NP was calculated using the following formula: Brightness value of $\mathrm{NP} /$ brightness value of cerebrospinal fluid (20).

Raman spectroscopy. Frozen sections of NP samples were thawed completely at room temperature and the OCT was then thoroughly washed with physiological saline solution and subsequently scanned using Raman spectroscopy. During the Raman measurement, NP samples were kept hydrated with physiological saline solution. Raman measurements were performed using a Laser Micro-Raman Spectrometer (Thermo Scientific DXR Raman Microscope) at room temperature. Collection parameters were set as follows: Laser wavelength, $532 \mathrm{~nm}$ (DXR $532 \mathrm{~nm}$ LASER); microscope objective, 50X; laser power, $10.0 \mathrm{~mW}$; spot size, $1.1 \mu \mathrm{m}$; spectral range, 800-1,800 $\mathrm{cm}^{-1}$; grating, 900 lines $/ \mathrm{mm}$; resolution, $5 \mathrm{~cm}^{-1}$. The data were collected at 10 randomly chosen points on the surface of the NP samples (21-23). The time of exposure to obtain individual Raman spectra was $16 \mathrm{sec}$, with five scans taken. In order to acquire high-quality spectra, re-focusing was necessary.

Data were pre-processed with OMINIC 9.3.32 software (Thermo Fisher Scientific, Inc.). For baseline correction, a linear baseline was fit automatically to the whole spectral range and subtracted from each spectrum of the dataset. Gaussian/Lorentzian functions were used throughout the spectral fitting computation. Raman band data (intensity or area) were imported to Microsoft Excel 2010 and band intensity ratios were calculated.

Statistical analysis. Statistical analysis was performed using SPSS 16.0 software (SPSS, Inc.) and data are expressed as the mean \pm standard deviation. Statistically significant differences between groups were determined using one-way analysis of variance, followed by Tukey's test for multiple comparisons. The inter-observer agreements of the IDD were assessed with $\kappa$ 
statistics. The association between spectral data and Pfirrmann grades was evaluated using Spearman's correlation test. The association between the T2 signal intensity value and the relative content of proteoglycans was evaluated using bivariate correlations. The graphs were produced with GraphPad Prism 5.0 Software (GraphPad Software, Inc.). $\mathrm{P}<0.05$ was considered to indicate a statistically significant difference.

\section{Results}

Pfirrmann grading. Representative MRI images of different patients are provided in Fig. 1A. The $\kappa$ value of the first measurement was 0.734 , indicating a good interobserver agreement $(\mathrm{P}<0.001)$ and the $\kappa$ value of the second measurement was $0.774(\mathrm{P}<0.001)$. The grading results are listed in Table I: Grade III, $\mathrm{n}=10$; grade IV, $\mathrm{n}=10$; and grade V, $\mathrm{n}=10$. None of the discs were graded as Pfirrmann grade I or II.

Analysis of the T2 signal intensity value. The T2 signal intensity value in patients with Pfirrmann grade IV and V was significantly lower compared with that in grade III $(\mathrm{P}<0.001)$; however, the T2 signal intensity value was not significantly different between grade IV and grade V $(\mathrm{P}>0.05)$. Spearman's rank correlation analysis demonstrated that the T2 signal intensity value was significantly inversely correlated with the Pfirrmann grade (r=-0.7967, P<0.0001; Fig. 1B).

Characteristics of Raman spectra. The Raman spectra of the NP samples with different Pfirrmann grades are presented in Fig. 2 and a list of bands and corresponding assignments observed in the average spectra are listed in Table II. The proteoglycans peaked at $1,064-1,065 \mathrm{~cm}^{-1}$, which corresponded to the glycosaminoglycans, which were previously identified in the Raman spectra of chondroitin sulfate and proteoglycans (11). The peak of the phenylalanine ring breathing band was at $1,004 \mathrm{~cm}^{-1}$. This was used to calculate the relative content, as it is not sensitive to local chemical environment (24). Due to the irregularity of biological material surfaces influencing the band intensity, band intensity ratios and band area integrals were employed to display compositional and structural changes of proteoglycans and collagen in NP samples. These parameters provide information on the association between proteoglycans, collagen content and structure in NP samples. In the present study, the intensity ratio of the two peaks $\left(\mathrm{I}_{1064} / \mathrm{I}_{1004}\right)$ provided information about the relative content of proteoglycans (13).

Collagen serves a major structural role in the intervertebral disc, which may be detected as Raman bands arising from the backbone amide groups of collagens. Amide I and III bands are uniquely useful for collagen conformational analysis. Bands generated in the 1,200-1,300 and 1,600-1,700 $\mathrm{cm}^{-1}$ range were identified as amide III and I, respectively, and were previously identified in the Raman spectra of collagen (25-27). The intensity ratio of two peaks $\left(\mathrm{I}_{1670} / \mathrm{I}_{1640}\right.$; amide $\left.\mathrm{I}\right)$ may provide information on the relative content of disordered coil (random coil) vs. ordered coil ( $\alpha$-helix) $(13,28,29)$.

Raman analysis of proteoglycan and collagen content in NP samples. The relative content of proteoglycans $\left(\mathrm{I}_{1064} / \mathrm{I}_{1004}\right)$ in grade IV and V samples was significantly decreased compared with that in grade III $(\mathrm{P}<0.01$ and $\mathrm{P}<0.001$, respectively).
Table I. Level of lumbar disc herniation in Pfirrmann grades.

\begin{tabular}{lccccc}
\hline & \multicolumn{4}{c}{ Level of lumbar disc herniation } & \\
\cline { 2 - 5 } Grade & $\mathrm{L}_{1}-\mathrm{L}_{2}$ & $\mathrm{~L}_{3}-\mathrm{L}_{4}$ & $\mathrm{~L}_{4}-\mathrm{L}_{5}$ & $\mathrm{~L}_{5}-\mathrm{S}_{1}$ & Total \\
\hline III & 0 & 1 & 5 & 4 & 10 \\
IV & 0 & 1 & 6 & 3 & 10 \\
V & 0 & 0 & 7 & 3 & 10 \\
Total & 0 & 2 & 18 & 10 & 30 \\
\hline
\end{tabular}

Spearman's rank correlation analysis results demonstrated that the relative content of proteoglycans was significantly inversely correlated with the Pfirrmann grade $(\rho=-0.6462$; $\mathrm{P}<0.0001$; Fig. 3A).

Collagen content was mainly determined through the area integral under the Raman spectral curve of the amide group bands. The content of amide III in grade IV was significantly increased compared with that in grade III and V $(\mathrm{P}<0.001$ and $\mathrm{P}<0.01$, respectively). Spearman's rank correlation analysis demonstrated that the content of amide III was not significantly correlated with the Pfirrmann grade $(\rho=0.2689$; $\mathrm{P}>0.05$; Fig. 3B). The content of amide I in grade IV was higher compared with that in grade III and V (Fig. 3C), suggesting it had reached its maximum at grade IV. Spearman's rank correlation analysis demonstrated that the content of amide I was significantly positively correlated with the Pfirrmann grade $(\rho=0.5141 ; P<0.01)$.

Raman analysis of collagen structural changes in NP samples. The intensity ratio of two peaks $\left(\mathrm{I}_{1670} / \mathrm{I}_{1640}\right.$; amide I) in grade IV and $\mathrm{V}$ was significantly higher compared with that in grade III $(\mathrm{P}<0.05$ and $\mathrm{P}<0.01$, respectively). Spearman's rank correlation analysis demonstrated that the intensity ratio of two peaks $\left(\mathrm{I}_{1670} / \mathrm{I}_{1640}\right.$; amide I) was significantly positively correlated with the Pfirrmann grade $(\rho=0.5330 ; \mathrm{P}<0.01$; Fig. $3 \mathrm{D})$.

\section{Discussion}

To the best of our knowledge, the present study was among the first to use Raman spectroscopy to report compositional and structural variations of the NP from patients with LDH. In the present study, $30 \mathrm{NP}$ tissue samples were obtained from 30 patients who were diagnosed with $\mathrm{LDH}$ and received spinal fusion surgery to relieve LBP. The Pfirrmann grading system, a semi-quantitative assessment, was used to evaluate the level of IDD according to the changes in the MRI signal intensity, the disc structure and the distinction between NP and annulus fibrosus (AF) and disc height. Although the Pfirrmann grade is widely applied in clinical practice worldwide, this classification system is subjective and ambiguous due to the lack of a quantitative index (10). It was observed that the interobserver agreements of the IDD displayed $\kappa_{1}=0.734$ and $\kappa_{2}=0.774$, hence the result is apparently acceptable. At present, a series of quantitative MRI diagnostic methods and quantitative MR parameters, including apparent diffusion coefficient and diffusion-weighted imaging, are used to quantitatively explore the process of IDD and to detect imperceptible biochemical 
Table II. Band position and assignments of selected Raman bands from ex vivo human nucleus pulposus tissue, using the interval $800-1,800 \mathrm{~cm}^{-1}$.

\begin{tabular}{llll}
\hline Band position $\left(\mathrm{cm}^{-1}\right)$ & \multicolumn{1}{c}{ Assignments } & Chemical compounds \\
\hline 1,004 & Phenylalanine & Protein & $($ Refs.) \\
1,064 & $\mathrm{OSO}_{3}^{-}$stretching; glycoaminoglycan & Proteoglycan & $(24)$ \\
$1,200-1,300$ & Amide III, major collagen band & Protein & $(11)$ \\
1,640 & Amide I, ordered coil, $\alpha$-helix & Protein & $(13,28,29)$ \\
1,670 & Amide I, disordered coil, random coil & Protein & $(13,28,29)$ \\
$1,600-1,700$ & Amide I, major collagen band & Protein & $(25-27)$ \\
\hline
\end{tabular}
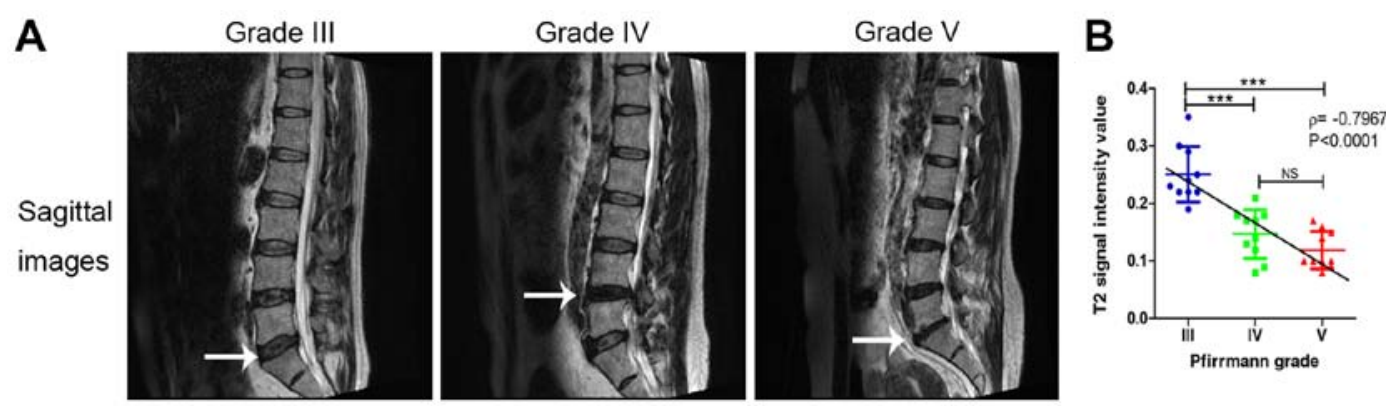

Figure 1. (A) Representative MRI scans of IDD in different Pfirrmann grades. Sagittal T2-weighted images of lumbar spines with different Pfirrmann grades. The white arrows indicate the location of impaired intervertebral discs. (B) Comparison of the T2 signal intensity value in different Pfirrmann grades. Spearman's rank correlation analysis demonstrated that the T2 signal intensity value was significantly inversely correlated with the Pfirrmann grade.

${ }^{* * *} \mathrm{P}<0.001$. NS, no significance; IDD, intervertebral disc degeneration.

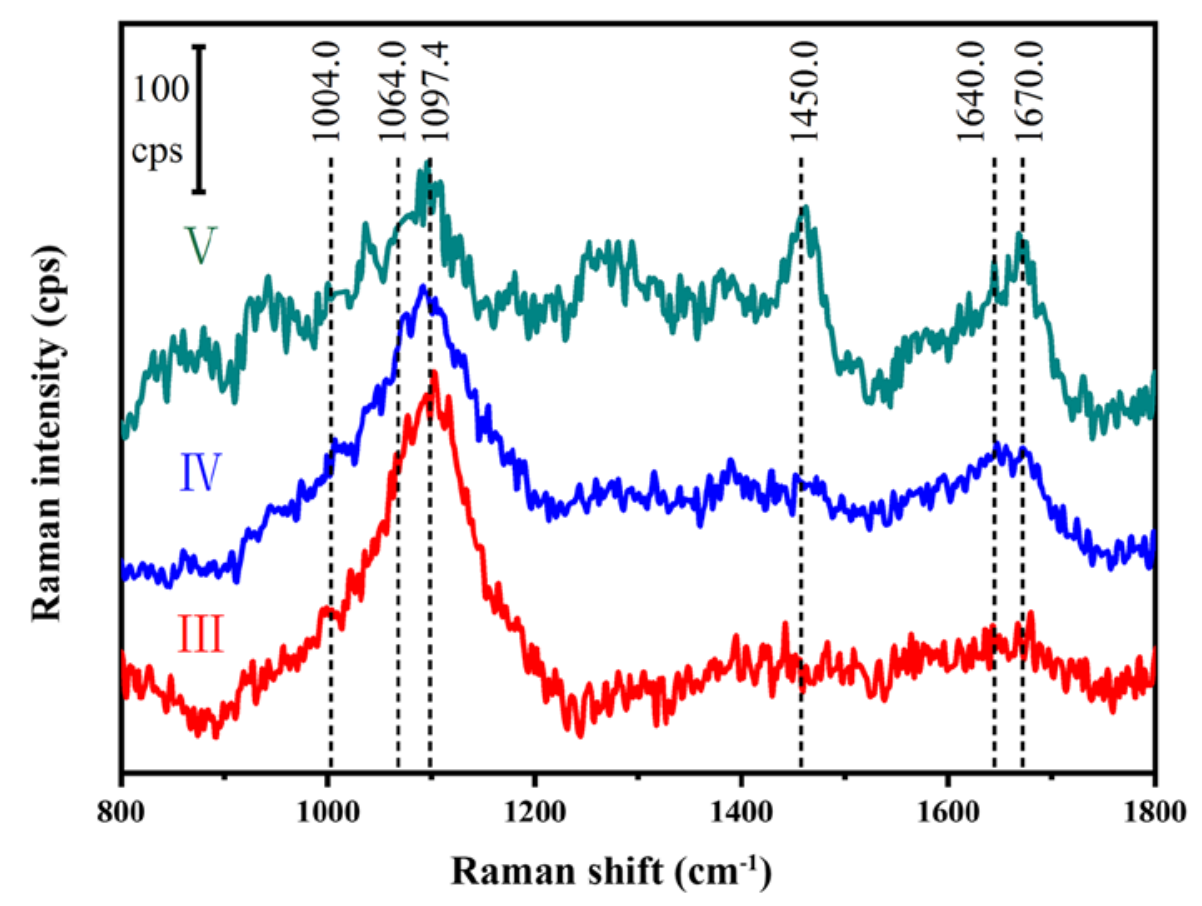

Figure 2. Raman spectra of the nucleus pulposus samples with Pfirrmann grades. Cps, counts per second.

changes within the discs. However, the intrinsic association between these quantitative MRI parameters and the biological properties of the intervertebral disc has remained to be completely elucidated. In the present study, the association between the $\mathrm{T} 2$ signal intensity value and the relative content of proteoglycans was evaluated using bivariate correlations. The results provided $\mathrm{P}=0.509$ and $\mathrm{P}<0.01$; therefore, their correlation was significant to the 0.01 level (two-tailed). This result indicated that the $\mathrm{T} 2$ signal intensity value obtained by MRI is identical to the relative content of proteoglycans 

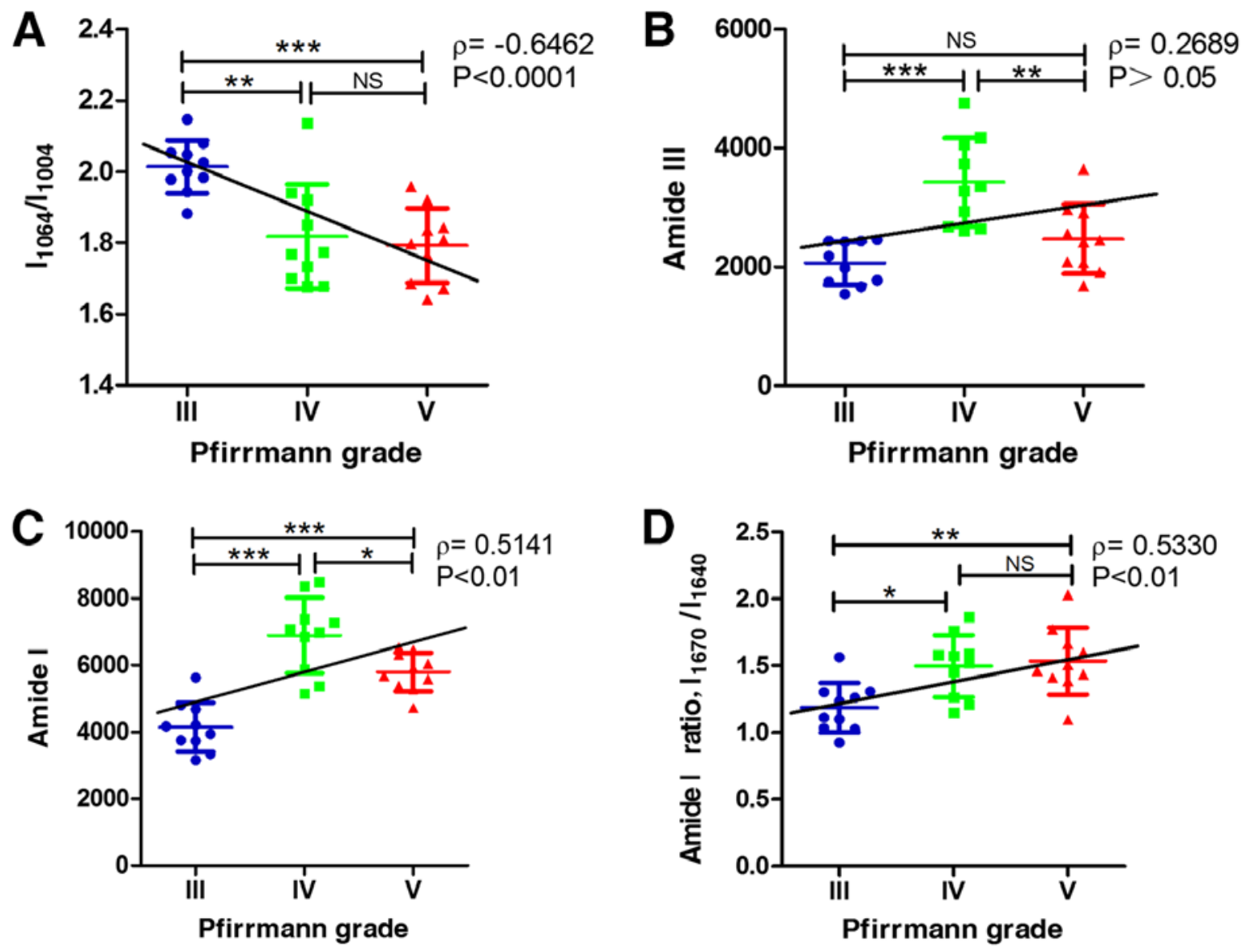

Figure 3. Raman spectroscopic analysis of proteoglycans and collagen content in NP samples. (A) Comparison of the relative content of proteoglycans $\left(\mathrm{I}_{1064} / \mathrm{I}_{1004}\right)$ among different Pfirrmann grades. Spearman's rank correlation analysis demonstrated that the relative content of proteoglycans $\left(\mathrm{I}_{1064} /\right.$ $\mathrm{I}_{1004}$ ) was significantly inversely correlated with the Pfirrmann grade. (B) Comparison of the content of collagen (Amide III) in different Pfirrmann grades. Spearman's rank correlation analysis demonstrated that the content of Amide III was not significantly correlated with the Pfirrmann grade. (C) Comparison of the content of collagen (Amide I) among different Pfirrmann grades. Spearman's rank correlation analysis demonstrated that the content of Amide I was significantly positively correlated with the Pfirrmann grade. (D) Comparison of the intensity ratio of two peaks ( $\mathrm{I}_{1670} / \mathrm{I}_{1640}$; Amide I) among different Pfirrmann grades. Spearman's rank correlation analysis results demonstrated that the intensity ratio of the two peaks ( $\mathrm{I}_{1670} /$ $\mathrm{I}_{1640}$; Amide I) was significantly positively correlated with the Pfirrmann grade. ${ }^{*} \mathrm{P}<0.05,{ }^{* *} \mathrm{P}<0.01,{ }^{* * * *} \mathrm{P}<0.001$. I, relative intensity; NS, no significance; $\mathrm{NP}$, nucleus pulposus.

$\left(\mathrm{I}_{1064} / \mathrm{I}_{1004}\right)$ determined using a Raman spectrometer. On the other hand, Yang et al and Benneker et al $(30,31)$ reported that the decreased content of proteoglycans in the NP was responsible for the decrease in the osmotic pressure of the disc matrices and loss of hydration. These results lead to the loss of signal intensity on T2-weighted MRI images. Unfortunately, at present, lumbar MRI is unable to display changes in collagen content and structure.

Raman spectroscopy is able to not only measure the chemical composition of complex biological samples, including biofluids, cells and tissues, but it also provides quantitative information about its chemical makeup. Subtle vibrational changes in biological macromolecule content and structure may be detected by Raman spectroscopy (12). Type II collagen and proteoglycans are the predominant components of the NP and decreased content of these two components is the major characteristic of IDD (19). In the present study, the relative content of proteoglycans $\left(\mathrm{I}_{1064} / \mathrm{I}_{1004}\right)$ demonstrated a significant negative correlation with the Pfirrmann grade. Thus, the relative content of proteoglycans decreased whilst the Pfirrmann grade increased, which may be responsible for the loss of hydration and the T2 signal intensity value of the NP, as the changes in signal intensity caused by the variations in tissue moisture content have been indicated to be most sensitive on T2WI $(32,33)$.

In the intervertebral disc, collagen serves a major structural role, particularly at the NP where type IX collagen fibers are cross-linked to collagen type II fibers to provide optimal stability (34). Collagen may be detected as Raman bands arising from the backbone amide groups in collagen. In the present study, Raman spectral analysis demonstrated that the content of amide III and I in grade IV was significantly higher compared with that in grade III and V; however, it reached its maximum at grade IV, instead of continuing to increase with the Pfirrmann grade. Furthermore, Spearman's rank correlation analysis demonstrated that the content of amide I was significantly positively correlated with the Pfirrmann grade. This transition into a more fibrotic type of tissue may produce a stiffer NP and the 'shock-absorbing' properties of the disc may be severely limited, eventually contributing to IDD. Decreased content of type II collagen and proteoglycans are the major features of IDD; however, the present study observed that 
the content of collagen increased with the Pfirrmann grade, which may contribute to metabolic disorders of the extracellular matrix. It has been reported that if the boundary between the outer AF and inner NP is disrupted, this may impair the mechanical function of the intervertebral disc (35).

Raman spectroscopy is not only able to identify compositional changes in collagen, but it is also sensitive to different protein amide linkages in the secondary structures, including $\alpha$-helices, $\beta$-sheets and random coils $(22,36)$. Amide III and I bands are uniquely useful for collagen conformational analysis. The relative content of disordered coil vs. ordered coil helps define the degree of disorder in the collagen secondary structure. Kumar et al (13) reported that the intensity ratio of two peaks $\left(\mathrm{I}_{1245} / \mathrm{I}_{1270}\right)$ provided information about the relative content of random vs. ordered coil in the protein structure and the progression of the cartilage disorder. However, in the present study, it was discovered that the intensity ratio of two peaks $\left(\mathrm{I}_{1670} / \mathrm{I}_{1640}\right.$; amide I) was significantly positively correlated with the Pfirrmann grade. The higher relative intensity ratio of $\mathrm{I}_{1670} / \mathrm{I}_{1640}$ indicates a higher fractional content of disordered collagen, which is in turn evidence for the defective collagen structure leading to abnormalities of the NP. These results may contribute to the further study of the microcosmic mechanisms of IDD and not only suggested an increase in the content of defective collagen with the Pfirrmann grade, but they also illustrated the ability of Raman spectroscopy to detect minute modifications in the NP at the microscopic level.

There are certain limitations to the present study. Although the content of proteoglycans and collagen was measured though band intensity and band area ratios, the relatively high background in the Raman spectra was unavoidable. Due to the irregularity of the biological material surfaces being able to significantly influence the band intensity and background (23), the same problem exists in other Raman spectroscopy experiments; for instance, in human knee cartilage, mesenchymal stromal cells and human brain tumors (21-23). In addition, 10 randomly selected points on the surface of each NP sample were analyzed by Raman spectrometry. It appears to be difficult to cover the entire NP. However, Tsao et al (21) reported that Raman spectra were obtained from at least 10 locations selected from the surface of cell samples for each sample preparation, Takahashi et al (22) reported that Raman spectral intensity at 10 different locations was randomly recorded within each of the total 6 areas labeled on each sample and Buchwald et al (23) reported that 7 Raman maps were measured in each defined central site of the femur head. Therefore, future studies should increase the scanning numbers and points for generating spectra with high quality.

In conclusion, the results of the present study provided a step towards the potential use of ex vivo Raman spectroscopy for the investigation of biomarkers in IDD. A higher relative intensity of the ratio of two peaks $\left(\mathrm{I}_{1670} / \mathrm{I}_{1640}\right.$; amide I) indicated a higher fractional content of disordered collagen, which provides evidence of the defective collagen structure leading to abnormalities of the NP. In the near future, it is hypothesized that ex vivo Raman spectroscopy or in vivo studies of the NP will not only aid in investigating the biochemical mechanisms of IDD at the microscopic level, but they will also be combined with other MRI parameters and fiber-optic probes to aid in the early diagnosis of IDD.

\section{Acknowledgements}

Not applicable.

\section{Funding}

No funding was received.

\section{Availability of data and materials}

The datasets used and/or analyzed during the present study are available from the corresponding author on reasonable request.

\section{Authors' contributions}

TaZ and ZLi designed the study and revised the manuscript; XW and JM performed the experiments and drafted the manuscript; XW and ZLia collected human NP tissue samples;QS and ToZ were responsible for measuring the T2 signal intensity value and evaluating the degree of IDD according to Pfirrmann grades; and JM, ZLi and WWL contributed to performing the measurements and analyzing the data obtained using Raman spectroscopy. All authors read and approved the final manuscript.

\section{Ethics approval and consent to participate}

The present study was approved by the Ethics Review Board of Tianjin First Central Hospital (Tianjin China).

\section{Patient consent for publication}

Patients consented to the publication of their MRI scanning images.

\section{Competing interests}

The authors declare that they have no competing interests.

\section{References}

1. Deyo RA and Mirza SK: Clinical practice. Herniated lumbar intervertebral disk. N Engl J Med 374: 1763-1772, 2016.

2. Kepler CK, Ponnappan RK, Tannoury CA, Risbud MV and Anderson DG: The molecular basis of intervertebral disc degeneration. Spine J 13: 318-330, 2013.

3. Kallewaard JW, Geurts JW, Kessels A, Willems P, van Santbrink H and van Kleef M: Efficacy, safety, and predictors of intradiscal methylene blue injection for discogenic low back pain: Results of a multicenter prospective clinical series. Pain Pract 16: 405-412, 2016

4. Cunha C, Silva AJ, Pereira P, Vaz R, Goncalves RM and Barbosa MA: The inflammatory response in the regression of lumbar disc herniation. Arthritis Res Ther 20: 251, 2018.

5. Cheung KM, Karppinen J, Chan D, Ho DW, Song YQ, Sham P, Cheah KS, Leong JC and Luk KD: Prevalence and pattern of lumbar magnetic resonance imaging changes in a population study of one thousand forty-three individuals. Spine (Phila $\mathrm{Pa}$ 1976) 34: 934-940, 2009.

6. Wang Y, Wang H, Lv F, Ma X, Xia X and Jiang J: Asymmetry between the superior and inferior endplates is a risk factor for lumbar disc degeneration. J Orthop Res 36: 2469-2475, 2018.

7. He A, Wang WZ, Qiao PF, Qiao GY, Cheng H and Feng PY: Quantitative evaluation of compressed 14-5 and s1 nerve roots of lumbar disc herniation patients by diffusion tensor imaging and fiber tractography. World Neurosurg 115: e45-e52, 2018. 
8. Zhang W, Ma X, Wang Y, Zhao J, Zhang X, Gao Y and Li S: Assessment of apparent diffusion coefficient in lumbar intervertebral disc degeneration. Eur Spine J 23: 1830-1836, 2014.

9. Radek M, Pacholczyk-Sienicka B, Jankowski S, Albrecht $Ł$, Grodzka M, Depta A and Radek A: Assessing the correlation between the degree of disc degeneration on the Pfirrmann scale and the metabolites identified in HR-MAS NMR spectroscopy. Magn Reson Imaging 34: 376-380, 2016.

10. Xiong X, Zhou Z, Figini M, Shangguan J, Zhang Z and Chen W: Multi-parameter evaluation of lumbar intervertebral disc degeneration using quantitative magnetic resonance imaging techniques. Am J Transl Res 10: 444-454, 2018.

11. Pudlas M, Brauchle E, Klein TJ, Hutmacher DW and Schenke-Layland K: Non-invasive identification of proteoglycans and chondrocyte differentiation state by Raman microspectroscopy. J Biophotonics 6: 205-211, 2013.

12. Kong K, Kendall C, Stone N and Notingher I: Raman spectroscopy for medical diagnostics-from in-vitro biofluid assays to in-vivo cancer detection. Adv Drug Deliv Rev 89: 121-134, 2015.

13. Kumar R, Singh GP, Gronhaug KM, Afseth NK, de Lange Davies C, Drogset JO and Lilledahl MB: Single cell confocal Raman spectroscopy of human osteoarthritic chondrocytes: A preliminary study. Int J Mol Sci 16: 9341-9353, 2015.

14. Keating ME and Byrne HJ: Raman spectroscopy in nanomedicine: Current status and future perspective. Nanomedicine (Lond) 8: 1335-1351, 2013.

15. Nima ZA, Biswas A, Bayer IS, Hardcastle FD, Perry D, Ghosh A Dervishi E and Biris AS: Applications of surface-enhanced Raman scattering in advanced bio-medical technologies and diagnostics. Drug Metab Rev 46: 155-175, 2014.

16. Gong B, Oest ME, Mann KA, Damron TA and Morris MD Raman spectroscopy demonstrates prolonged alteration of bone chemical composition following extremity localized irradiation. Bone 57: 252-258, 2013

17. Lu FK, Calligaris D, Olubiyi OI, Norton I, Yang W, Santagata S Xie XS, Golby AJ and Agar NY: Label-free neurosurgical pathology with stimulated Raman imaging. Cancer Res 76 : 3451-3462, 2016.

18. Pence I and Mahadevan-Jansen A: Clinical instrumentation and applications of Raman spectroscopy. Chem Soc Rev 45: 1958-1979, 2016

19. Wang WJ, Yang W, Ouyang ZH, Xue JB, Li XL, Zhang J, He WS, Chen WK, Yan YG and Wang C: MiR-21 promotes ECM degradation through inhibiting autophagy via the PTEN/akt/mTOR signaling pathway in human degenerated NP cells. Biomed Pharmacother 99: 725-734, 2018.

20. Luoma EK, Raininko R, Nummi PJ, Luukkonen R, Manninen HI and Riihimäki HA: Suitability of cerebrospinal fluid as a signal-intensity reference on MRI: Evaluation of signal-intensity variations in the lumbosacral dural sac. Neuroradiology 39: 728-732, 1997.

21. Tsao YT,Huang YJ,Wu HH,Liu YA,Liu YS and Lee OK: Osteocalcin mediates biomineralization during osteogenic maturation in human mesenchymal stromal cells. Int J Mol Sci 18: pii: E159, 2017.

22. Takahashi Y, Sugano N, Takao M, Sakai T, Nishii T and Pezzotti G: Raman spectroscopy investigation of load-assisted microstructural alterations in human knee cartilage: Preliminary study into diagnostic potential for osteoarthritis. J Mech Behav Biomed Mater 31: 77-85, 2014

23. Buchwald T, Niciejewski K, Kozielski M, Szybowicz M, Siatkowski $M$ and Krauss H: Identifying compositional and structural changes in spongy and subchondral bone from the hip joints of patients with osteoarthritis using Raman spectroscopy. J Biomed Opt 17: 017007, 2012.
24. Caraher MC, Sophocleous A, Beattie JR, O'Driscoll O, Cummins NM, Brennan O, O'Brien FJ, Ralston SH, Bell SEJ, Towler M and Idris AI: Raman spectroscopy predicts the link between claw keratin and bone collagen structure in a rodent model of oestrogen deficiency. Biochim Biophys Acta Mol Basis Dis 1864: 398-406, 2018.

25. Quade M, Schumacher M, Bernhardt A, Lode A, Kampschulte M, Voß A, Simon P, Uckermann O, Kirsch M and Gelinsky M: Strontium-modification of porous scaffolds from mineralized collagen for potential use in bone defect therapy. Mater Sci Eng C Mater Biol Appl 84: 159-167, 2018.

26. Murab S and Ghosh S: Impact of osmoregulatory agents on the recovery of collagen conformation in decellularized corneas. Biomed Mater 11: 065005, 2016.

27. Masic A, Bertinetti L, Schuetz R, Galvis L, Timofeeva N, Dunlop JW, Seto J, Hartmann MA and Fratzl P: Observations of multiscale, stress-induced changes of collagen orientation in tendon by polarized Raman spectroscopy. Biomacromolecules 12: 3989-3996, 2011

28. Unal M, Jung H and Akkus O: Novel Raman spectroscopic biomarkers indicate that postyield damage denatures bone's collagen. J Bone Miner Res 31: 1015-1025, 2016.

29. Lurie JD, Moses RA, Tosteson AN, Tosteson TD, Carragee EJ, Carrino JA, Kaiser JA and Herzog RJ: Magnetic resonance imaging predictors of surgical outcome in patients with lumbar intervertebral disc herniation. Spine (Phila Pa 1976) 38: 1216-1225, 2013

30. Yang SH, Espinoza Orias AA, Pan CC, Senoo I, Andersson GBJ, An HS and Inoue N: Spatial geometric and magnetic resonance signal intensity changes with advancing stages of nucleus pulposus degeneration. BMC Musculoskelet Disord 18: 473, 2017.

31. Benneker LM, Heini PF, Anderson SE, Alini M and Ito K: Correlation of radiographic and MRI parameters to morphological and biochemical assessment of intervertebral disc degeneration. Eur Spine J 14: 27-35, 2005.

32. Cassinelli EH, Hall RA and Kang JD: Biochemistry of intervertebral disc degeneration and the potential for gene therapy applications. Spine J 1: 205-214, 2001.

33. Tertti M,Pajanen H, Laato M, Aho H, Komu M and Kormano M: Disc degeneration in magnetic resonance imaging. A comparative biochemical, histologic, and radiologic study in cadaver spines. Spine (Phila Pa 1976) 16: 629-634, 1991.

34. Kalb S, Martirosyan NL, Kalani MY, Broc GG and Theodore N: Genetics of the degenerated intervertebral disc. World Neurosurg 77: 491-501, 2012.

35. Shi C, Wu H, Du D, Im HJ, Zhang Y, Hu B, Chen H, Wang X, Liu Y, Cao $\mathrm{P}$, et al: Nicotinamide phosphoribosyltransferase inhibitor APO866 prevents IL-1 $\beta$-induced human nucleus pulposus cell degeneration via autophagy. Cell Physiol Biochem 49: 2463-2482, 2018.

36. Dehring KA, Smukler AR, Roessler BJ and Morris MD: Correlating changes in collagen secondary structure with aging and defective type II collagen by Raman spectroscopy. Appl Spectrosc 60: 366-372, 2006.

This work is licensed under a Creative Commons Attribution-NonCommercial-NoDerivatives 4.0 International (CC BY-NC-ND 4.0) License. 